\title{
El ejercicio físico en el adulto mayor obeso
}

\author{
Physical exercise in the obese older adult
}

Efraín Velasteguí López. ${ }^{1}$ \& Lic. Joel García Espinosa. ${ }^{2}$

DOI: https://doi.org/10.33262/anatomiadigital.v1i3.1125

\begin{abstract}
.
A descriptive, longitudinal study was carried out on a group of elderly people with obesity in the municipality of Melena del Sur, Mayabeque province, which is located in the area belonging to the Basic Health Team No. 1 of the Raúl Curras Regalado Polyclinic, with the objective of designing a proposal for physical exercises that contribute to improving the participation of obese older adults between the ages of 65 and 75, for 3 months, in a period of time from January to March of the year 2020. The study will participate 12 obese older adults, 7 of them are women for $58.33 \%$ and 5 are men for $41.66 \%$.
\end{abstract}

Keywords: descriptive, longitudinal study.

\section{Resumen.}

Se realizó un estudio descriptivo, longitudinal, a un grupo de personas de la tercera edad con obesidad del municipio de Melena del Sur, provincia Mayabeque, que se encuentra en la zona perteneciente al Equipo Básico de Salud No 1 del Policlínico Raúl Curras Regalado, con el objetivo de diseñar una propuesta de ejercicios físicos que contribuyan a mejorar la participación de los adultos mayores obesos en edades comprendidas entre 65 y 75 años, durante 3 meses, en un período de tiempo comprendido de Enero a Marzo del año 2020. En el estudio participarán 12 adultos mayores obesos, de ellos 7 son mujeres para un $58.33 \%$ y 5 son hombres para un $41.66 \%$.

Palabras claves: Estudio descriptivo longitudinal.

1 Ciencia digital, Ambato, Ecuador, luisefrainvelastegui@cienciadigital.org https://orcid.org/0000-0002-7353-0168

${ }^{2}$ Ciencias Médicas Mayabeque, Mayabeque, Cuba, jgarcia@infomed.sld.cu ID https://orcid.org/0000-00027353-0169 


\section{Introducción.}

Con el aumento creciente de la población mayor en el mundo, las sociedades están llamadas a desarrollar sistemas o programas de atención al adulto mayor, específicamente en el área de la actividad física, por la importancia, las ventajas y beneficios que conlleva la práctica de la misma.

Los adultos mayores son aquellas personas de 59 años en adelante, aunque también se alude a ellos como ancianos, viejitos, adultos en plenitud, tercera edad, juventud acumulada y senectud. El envejecimiento se clasifica según la Organización Panamericana de la Salud (OPS, 2012) como: tercera edad (aquellos que tienen 60 años), adulto mayor (más de 65 años) y cuarta edad (son los que tienen de 80 años en adelante).

El ejercicio físico es una actividad planificada, ordenada y repetitiva, que se lleva a cabo en los momentos de tiempo libre, en el cual se incluye el baile, deporte, gimnasia, educación física, entre otros. Este es sinónimo de bienestar físico, mental y social de una persona.

En nuestro país se realiza un intenso trabajo para ofrecer una mejor atención a la población en la eliminación de enfermedades, para de esta forma ayudar a una salud más eficaz. Actualmente se han creado áreas terapéuticas mancomunándose los esfuerzos del Ministerio de Salud Pública, el INDER y el Ministerio de Educación para brindar atención especial con ejercicios físicos específicos a aquellos que presenten alguna enfermedad o dolencia.

Los conocimientos acerca de la importancia que ha alcanzado hoy en día la práctica del ejercicio físico como medio del proceso de rehabilitación son importantes, donde millones de personas han sido atendidas y beneficiadas, logrando que aumente la calidad de vida, el fortalecimiento de los órganos y sistemas del organismo, reduciendo así los dolores y la elevación de las posibilidades funcionales que garantiza el desarrollo de las cualidades motoras mejorando la capacidad funcional del mismo.

En la provincia Mayabeque, considerando que el grupo de adultos mayores obesos es muy heterogéneo y con características diferentes, es necesario implementar programas que den respuesta a sus necesidades, que beneficien tanto a las personas adultas mayores estudiadas, como a las de la comunidad, dentro de un marco de atención con calidad y humanismo que les permita una vida plena.

En relación con lo planteado en los párrafos precedentes nos planteamos el siguiente problema de investigación: ¿Cómo incrementar la participación de los adultos mayores obesos al ejercicio físico en edades comprendidas entre 65 y 75 años del consultorio $\mathrm{N}^{\mathrm{o}} 1$ del municipio Melena del Sur, provincia Mayabeque?

\section{Desarrollo}

La expresión tercera edad es un término social que hace referencia a la población de personas mayores o ancianas, está siendo sustituido por el término personas mayores (en España) y adulto mayor (en América Latina). Esta etapa, también denominada vejez o ancianidad, es el 
último periodo de la vida, donde el cuerpo sufre un deterioro. Se trata de un grupo de población que tiene como mínimo 65 años de edad. A partir de esta edad aparecen, con mayor frecuencia un número creciente de enfermedades.

A la obesidad se le denomina como la acumulación excesiva y generalizada de grasa en el cuerpo, con hipertrofia total del tejido adiposo, produciendo cambios morfológicos, nutricionales y metabólicos. No es más que la alteración del balance energético de alimento y el poco gasto calórico donde se acumula el exceso en forma de grasa dando origen al sobrepeso y la obesidad.

Lo cierto es que esta alteración metabólica que es conocida desde la edad antigua, está muy de moda en los últimos tiempos, siendo una de las principales causas de la hipertensión arterial, la diabetes mellitus, litiasis biliar, insuficiencia coronaria y la arteriosclerosis entre otras enfermedades que se conocen como las principales causas de muerte.

\section{Diseño Metodológico}

Se realizó un estudio descriptivo, longitudinal, a un grupo de personas de la tercera edad con obesidad del municipio de Melena del Sur, que se encuentra en la zona perteneciente al Equipo Básico de Salud No 1 del Policlínico Raúl Curras Regalado, con el objetivo de diseñar una propuesta de ejercicios físicos que contribuyan a mejorar la participación de los adultos mayores obesos en edades comprendidas entre 65 y 75 años, durante 3 meses, en un período de tiempo comprendido de Enero a Marzo del año 2020.

\section{Universo y muestra}

El universo poblacional del consultorio $\mathrm{N}^{\mathrm{o}} 1$ del Municipio Melena del Sur está representado por 22 adultos mayores entre 65-75 años. Como muestra 12 adultos mayores son obesos, de ellos 7 son mujeres para un $58.33 \%$ y 5 son hombres para un $41.66 \%$, los mismos se escogieron debido a su poca motivación para realizar ejercicios con el fin de mejorar su salud y el riesgo de contraer enfermedades no trasmisibles en la tercera edad. De los 12 obesos, además de su patología 3 son hipertensos, 4 presentan diabetes mellitus, 3 padecen artrosis y 2 sufren de cardiopatía isquémica. De ellos 6 fuman, 2 ingieren bebidas alcohólicas y los 12manifiestan hábitos alimentarios negativos para su salud. Anexo 1.

\section{Criterio de inclusión:}

- Hombre o mujer de 65 a 75 años.

- Firmar el consentimiento informado, documento donde se explica el procedimiento del programa de ejercicios propuestos por la investigación

\section{Criterios de exclusión:}


- Contraindicaciones para realizar ejercicios.

- Sordera y ceguera.

- Residente con enfermedad terminal.

- Haber sido operado quirúrgicamente en los dos meses previos al estudio.

La población en estudio,65 a 75 años, muestra que en relación al IMC,hay diferencias significativas entre hombres y mujeres, siendo más elevada en éstas, de ellas 3 tienen sobrepeso (25\%) y 4 son obesas (33.33)\% y de los hombres 2 tienen sobrepeso (16.66\%) y 3 son obesos (25\%).Anexo 2.

\section{Material y métodos}

- Cinta métrica

- Peso

- Talla

- Índice de masa corporal (imc), permite cuantificar la obesidad y la clasificación del individuo. Se calcula de la siguiente forma: imc $=(\mathrm{kg} / \mathrm{m} 2)$.

- Peso saludable (ps)

- Estetoscopio y esfigmomanómetro

Propuesta de ejercicio físico que contribuya a mejorar la participación de los adultos mayores obesos en edades comprendidas entre 65 y 75 años:

Tabla 1 Propuesta de ejercicio físico

\begin{tabular}{|c|c|c|c|c|c|}
\hline Ejercicio & Duración & Intensidad & F/card. & Frecuencia & Período \\
\hline Caminar & 30 min & $\begin{array}{c}60 \% \text { del } \\
\mathrm{VO}_{2} \text { Máx. }\end{array}$ & Mínima $103 \mathrm{~L} / \mathrm{min}$ & $\begin{array}{c}3 \text { días a la } \\
\text { semana }\end{array}$ & 6 semanas \\
\hline Caminar & $40 \mathrm{~min}$ & $\begin{array}{c}65 \% \text { del } \\
\mathrm{VO}_{2} \text { Máx. }\end{array}$ & $\begin{array}{c}\text { Mínima } 112 \mathrm{~L} / \mathrm{min} . \\
65 \% \mathrm{~F} / \mathrm{c}\end{array}$ & $\begin{array}{c}4 \text { días a la } \\
\text { semana }\end{array}$ & 6 semanas \\
\hline $\begin{array}{c}\text { Caminar/ } \\
\text { Trotar }\end{array}$ & $40 \mathrm{~min}$ & $\begin{array}{c}65 \% \text { del } \\
\mathrm{VO}_{2} \text { Máx. }\end{array}$ & $\begin{array}{c}\text { Máximo112 L/min } \\
65 \% \mathrm{~F} / \mathrm{c}\end{array}$ & $\begin{array}{c}4 \text { días a la } \\
\text { semana }\end{array}$ & 6 semanas \\
\hline $\begin{array}{c}\text { Trotar/ } \\
\text { Caminar }\end{array}$ & 50 min & $\begin{array}{c}70 \% \text { del } \\
\mathrm{VO}_{2} \text { Máx. }\end{array}$ & $\begin{array}{c}\text { Máximo } 120 \mathrm{~L} / \mathrm{min} \\
70 \% \mathrm{~F} / \mathrm{c}\end{array}$ & $\begin{array}{c}5 \text { días a la } \\
\text { semana }\end{array}$ & 6 semanas \\
\hline
\end{tabular}




\begin{tabular}{|c|c|c|c|c|c|}
$\begin{array}{c}\text { Trote muy } \\
\text { suave }\end{array}$ & $30 \mathrm{~min}$ & $\begin{array}{c}75 \% \text { del } \\
\mathrm{VO}_{2} \text { Máx. }\end{array}$ & Máximo $130 \mathrm{~L} / \mathrm{min}$ & $\begin{array}{c}6 \text { días a la } \\
\text { semana }\end{array}$ & 6 semanas \\
\hline
\end{tabular}

Fuente: Elaboración propia.

Gráfico 1 Distribución de las personas obesas de 65 a 75 años acompañada de otras patologías.

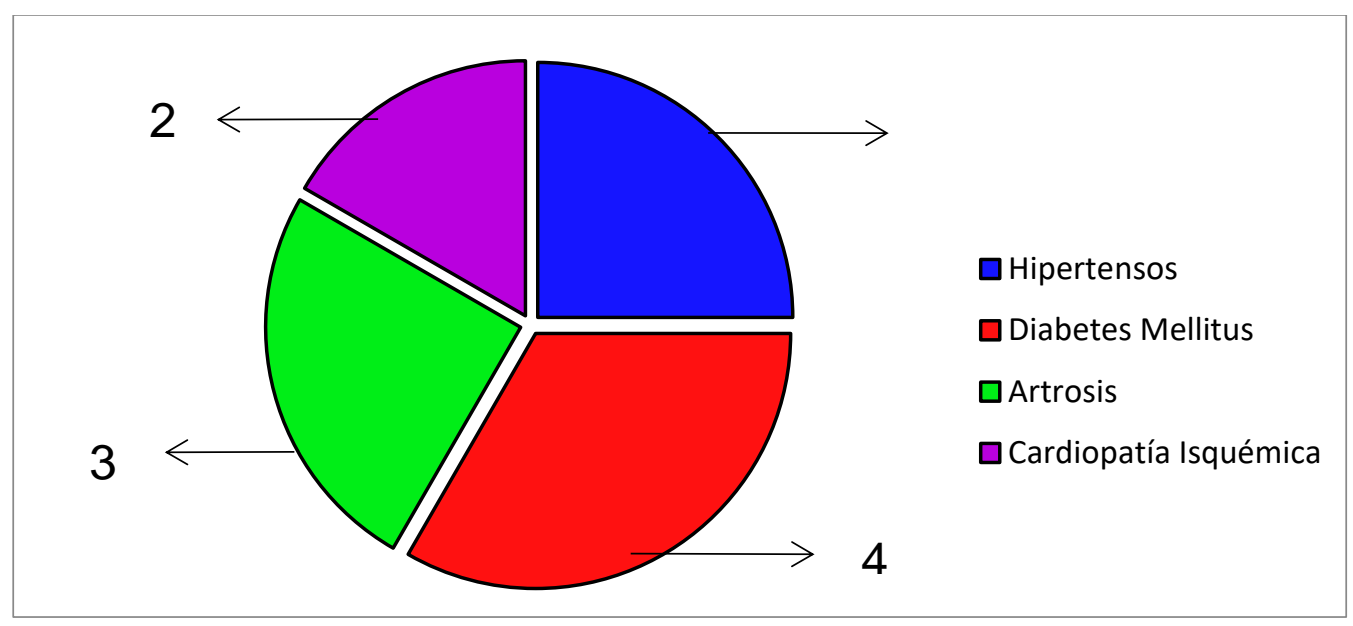

Fuente: Elaboración propia.

Gráfico N.2 Proporción adultos mayores con obesidad según el peso y el sexo.

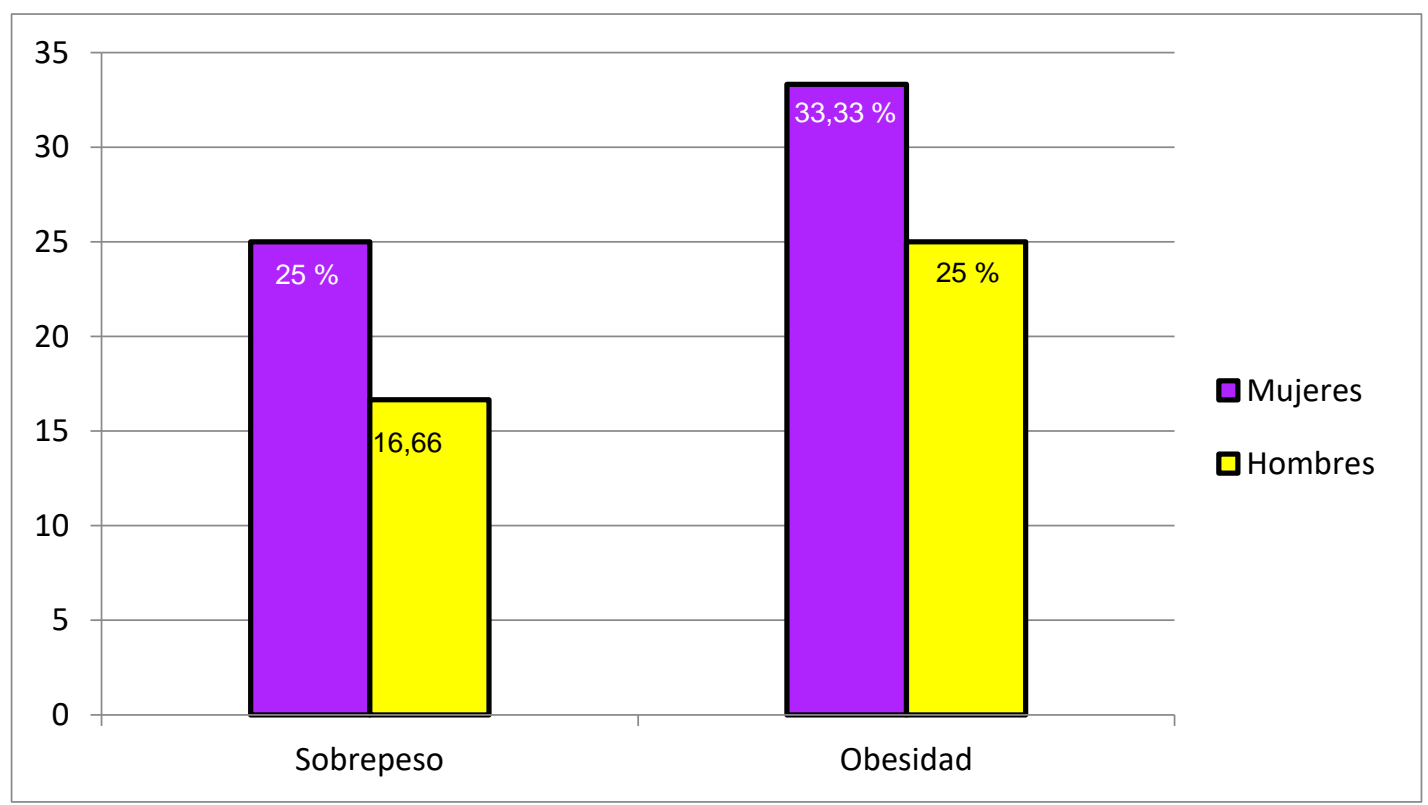

Fuente: Elaboración propia. 


\section{Conclusiones.}

- Con la investigación quisimos profundizar a cerca de los beneficios que traería la práctica de los ejercicios físicos en las personas de la tercera edad con obesidad.

- Brindar ejercicios físicos que contribuyan a mejorar la calidad de vida de la población obesa.

- El plan de acciones en función de la determinación de necesidades contempla acciones dirigidas a elevar los indicadores de incorporación de los adultos mayores obesos en el municipio de Melena del Sur.

\section{Referencias bibliográficas.}

Barroso Urrutia de Lourdes. Sociología y Trabajo Social aplicado. Ciudad de La Habana: Editorial Deportes, 2006--p15.

Colectivo de autores. Selección de Lecturas sobre Sociología y trabajo Social. Ciudad de La Habana: Editorial Deportes, 2006- p48.

Colectivo de autores. Selección de Lecturas sobre Sociología y trabajo Social. Ciudad de La Habana: Editorial Deportes, 2006-p8.

Colectivo de autores. Selección de Lecturas sobre Sociología y trabajo Social. Ciudad de La Habana: Editorial Deportes, 2006- p156.

Colectivo de autores. Selección de Lecturas sobre Sociología y trabajo Social. Ciudad de La Habana: Editorial Deportes, 2006- p156.

Colectivo de autores. Selección de Lecturas sobre Sociología y trabajo Social. Ciudad de La Habana: Editorial Deportes, 2006- p156.

American PsychologicalAssociation. (2010). Manual de publicaciones de la American PsychologicalAssociation (3ra ed.). México: Editorial el Manual Moderno S.A. de C.V

Aramburu y cols. (2000), la violencia en el noviazgo

Argyle, M., \& Henderson, M. (1985). The anatomy of relationships. London: Methuen.

Bardin, L. (1991) Análisis de contenido (vol.89). Ediciones Akal.

Bauman, y Zygmunt (2003). Individualmente pero juntos en Beck, Ulrich y Elizabeth BeckGernsheim. La individualización. El individualismo institucionalizado y sus consecuencias sociales y políticas. Paidos, pp.19. 
PARA CITAR EL ARTÍCULO INDEXADO.

Velasteguí López, E., \& García Espinosa, J. (2018). El ejercicio físico en el adulto mayor obeso. Anatomía Digital, 1(3), 55-61. https://doi.org/10.33262/anatomiadigital.v1i3.1125

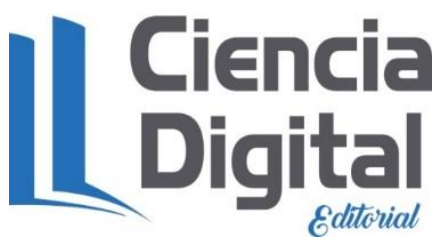

El artículo que se publica es de exclusiva responsabilidad de los autores y no necesariamente reflejan el pensamiento de la Revista Anatomía Digital.

El artículo queda en propiedad de la revista y, por tanto, su publicación parcial y/o total en otro medio tiene que ser autorizado por el director de la Revista Anatomía Digital.
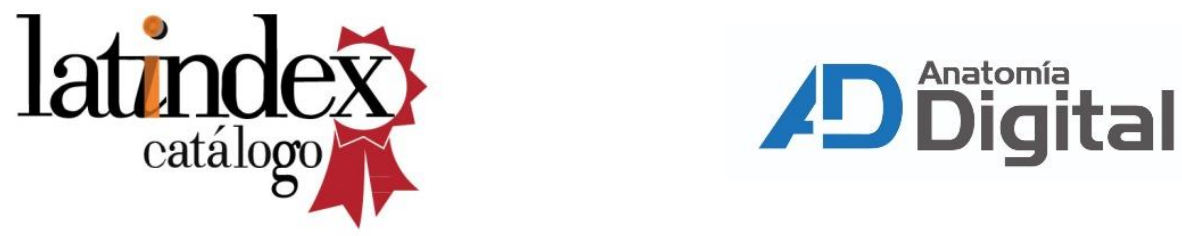